\title{
On topological properties of some convex polytopes by using line operator on their subdivisions
}

\author{
Fatima Asif ${ }^{1}(\mathbb{D})$, Zohaib Zahid ${ }^{1}(\mathbb{D})$, Sohail Zafar ${ }^{1}$ (D), Mohammad R. Farahani*2 ${ }^{(\mathbb{D}}$, \\ Wei $\mathrm{Gao}^{3}$ (D) \\ ${ }^{1}$ Department of Mathematics, University of Management and Technology (UMT), Lahore, Pakistan \\ ${ }^{2}$ Department of Applied Mathematics, Iran University of Science and Technology, Narmak, Tehran, \\ 16844, Iran \\ ${ }^{3}$ School of Information Science and Technology, Yunnan Normal University, Kunming, China
}

\begin{abstract}
In this paper, we give theoretical results for some topological indices such as Zagreb indices $M_{1}(G), M_{2}(G), M_{3}(G), R(G), M_{1}(\bar{G}), M_{2}(\bar{G})$, Zagreb coindices $\overline{M_{1}}(G), \overline{M_{2}}(G), \overline{M_{2}}(\bar{G})$ hyper-Zagreb index $H M(G)$, atom-bond connectivity index $A B C(G)$, sum connectivity index $\chi(G)$ and geometric-arithmetic connectivity index $G A(G)$, by considering $G$ as line graph of subdivision of some convex polytopes and $\bar{G}$ denotes its complement.
\end{abstract}

Mathematics Subject Classification (2010). 05C90, 05C35, 05C12

Keywords. Topological indices, line graph, subdivision, convex polytopes

\section{Introduction and preliminaries}

According to Trinajstić and Gutman, mathematical chemistry is that part of theoretical chemistry which is concerned with applications of mathematical methods to chemical problems. Chemical graph theory, a branch of mathematical chemistry, applies graph theory to mathematical modeling of chemical phenomena. A topological index is a numerical value associated with chemical constitution for correlation of chemical structure with various physical properties, chemical reactivity or biological activity [28].

Let $G$ be a connected graph with vertex set $V(G)$ and edge set $E(G) \subseteq V(G) \times V(G)$. Let $p=|V(G)|$, the order of $G$ and $q=|E(G)|$, the size of $G$. The complement of a graph $G$, denoted by $\bar{G}$, is a simple graph having same set of vertices $V(G)$ in which any two vertices that are connected by an edge, if and only if they are not adjacent in $G$. Let $K_{p}$ is complete graph of order $p$ then we obtained $E(G) \cup E(\bar{G})=E\left(K_{p}\right)$ and $|E(\bar{G})|=\frac{p(p-1)}{2}-q$. The degree $d_{v}$ of any vertex $v$ is defined as the number of vertices joining to that vertex $v$ and the degree $d_{e}$ of an edge $e \in E(G)$ is defined as the number of its adjacent vertices in $V(L(G)$ ), where $L(G)$ is the line graph whose vertices are the edges of $G$ and they are adjacent if and only if they have a common end point in $G$. In structural chemistry, line

\footnotetext{
*Corresponding Author.

Email addresses: fatimaasif@uoslahore.edu.pk (F. Asif), zohaib_zahid@hotmail.com(Z. Zahid), sohailahmad04@gmail.com (S. Zafar), mrfarahani88@gmail.com (M.R. Farahani) gaowei@ynnu.edu.cn (W. Gao)

Received: 26.07.2017; Accepted: 02.10.2018
} 
graph of a graph $G$ is very useful. The first topological index on the basis of line graph was introduced by Bertz in 1981 (see [4]). For more details on line graph see the articles $[11,13-15,18,22]$. The subdivision $S(G)$ of a graph $G$ can be obtained by replacing each edge of $G$ by a path of length 2 , or we can say by inserting an additional vertex between each pair of vertices of $G$. For more details on the topological indicies of $L(S(G))$ we refer to the articles [24-26]). Our goal is to compute topological Indices of line graph of subdivision of some convex polytopes. Convex polytopes are fundamental geometric objects. The beauty of their theory is nowadays complemented by their importance for many other mathematical subjects, ranging from integration theory, algebraic topology, and algebraic geometry to linear and combinatorial optimization (see [9]). Also people are paying attention in finding metric dimension and labeling of convex polytopes (see $[2,3,20,21])$. From these motivational work, we take a step in finding the topological indices of line graph of subdivision of some convex polytopes.

The following lemma is helpful for computing the degree of a vertex of line graph.

Lemma 1.1. Let $G$ be a graph with $u, v \in V(G)$ and $e=u v \in E(G)$. Then:

$$
d_{e}=d_{u}+d_{v}-2 .
$$

Lemma 1.2. [12] Let $G$ be a graph of order $p$ and size $q$, then the line graph $L(G)$ of $G$ is a graph of order $q$ and size $\frac{1}{2} M_{1}(G)-q$.

\section{Line graphs of subdivision of some convex polytopes}

In this section we will discuss the combinatorial aspects of subdivision of some convex polytopes and line graphs of their subdivisions.

\subsection{Convex polytope $D_{n}$}

The graph of convex polytope $D_{n}$ consists of 5 -sided faces and $n$-sided face as defined in [2]. The convex polytope $D_{n}$ for $n=8$ is shown in Figure 1.

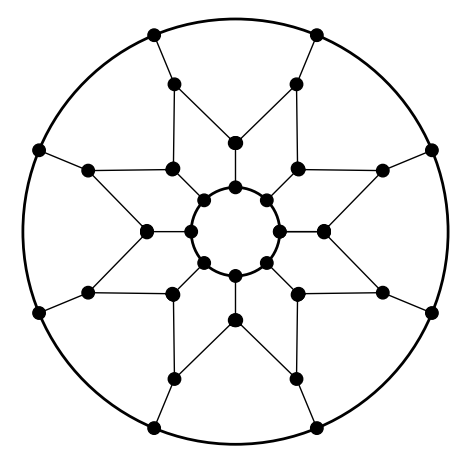

Figure 1. $D_{8}$

2.1.1. Subdivision of convex polytope $D_{n}$. We obtain the subdivision $S\left(D_{n}\right)$ by inserting an additional vertex between each pair of adjacent vertices of $D_{n}$. The subdivision of $D_{n}$ for $n=8$ is shown in Figure 2. The $S\left(D_{n}\right)$ consists of $10 n$ vertices out of which $6 n$ vertices are of degree 2 and $4 n$ vertices are of degree 3. Using Lemma 1.2, we have $\left|E\left(S\left(D_{n}\right)\right)\right|=18 n$. 


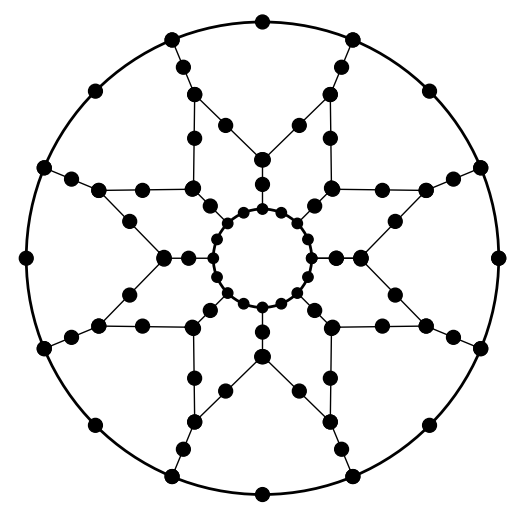

Figure 2. $S\left(D_{8}\right)$

2.1.2. Line graph of subdivision of convex polytope $D_{n}$. The line graph of subdivision of $D_{n}$ consists of $12 n$ vertices and all of them are of degree 3. Using Lemma 1.2, we have $\mid E\left(L\left(S\left(D_{n}\right)\right) \mid=18 n\right.$. The $L\left(S\left(D_{n}\right)\right)$ is shown in Figure 3 for $n=8$.

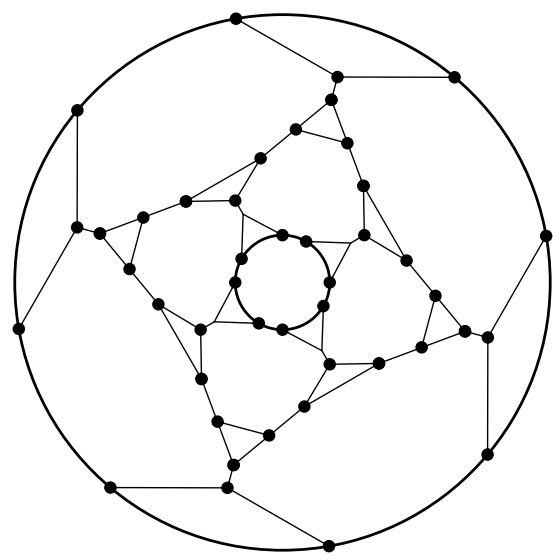

Figure 3. $L\left(S\left(D_{8}\right)\right)$

\subsection{Convex polytope $Q_{n}$}

The graph of convex polytope $Q_{n}$ consists of 3 -sided faces, 4 -sided faces, 5 -sided faces and $n$-sided face as defined in [3]. The convex polytope $Q_{n}$ for $n=8$ is shown in Figure 4. Both $D_{n}$ and $Q_{n}$ have same vertex set.

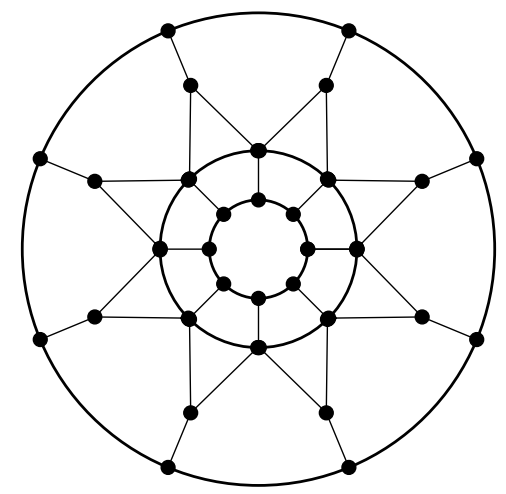

Figure 4. $Q_{8}$

2.2.1. Subdivision of convex polytope $Q_{n}$. We obtain the subdivision $S\left(Q_{n}\right)$ by inserting additional vertex between each pair of adjacent vertices of $Q_{n}$. The subdivision of $Q_{n}$ for $n=8$ is shown in Figure 5. The $S\left(Q_{n}\right)$ consists of $11 n$ vertices out which $7 n$ 
vertices are of degree $2,3 n$ vertices are of degree 3 and $n$ vertices are of degree 5 . Using Lemma 1.2, we have $\left|E\left(S\left(Q_{n}\right)\right)\right|=14 n$.

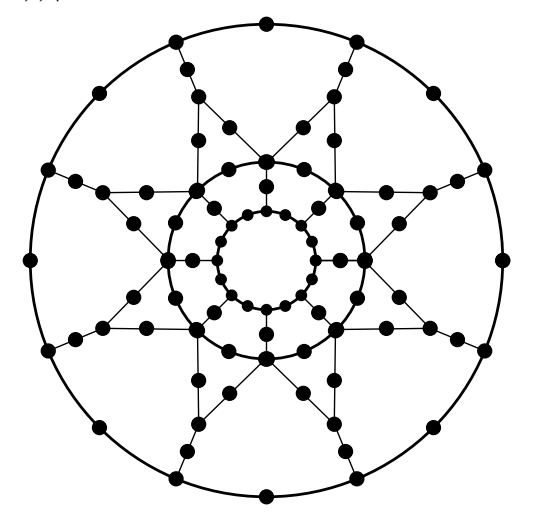

Figure 5. $S\left(Q_{8}\right)$

2.2.2. Line graph of subdivision of convex polytope $Q_{n}$. The line graph of subdivision of $Q_{n}$ consists of $14 n$ vertices out which $9 n$ vertices are of degree 3 and $5 n$ vertices are of degree 5 . Using Lemma 1.2, we have $\left|E\left(L\left(S\left(Q_{n}\right)\right)\right)\right|=26 n$. The $L\left(S\left(Q_{n}\right)\right)$ is shown in Figure 6 for $n=8$.

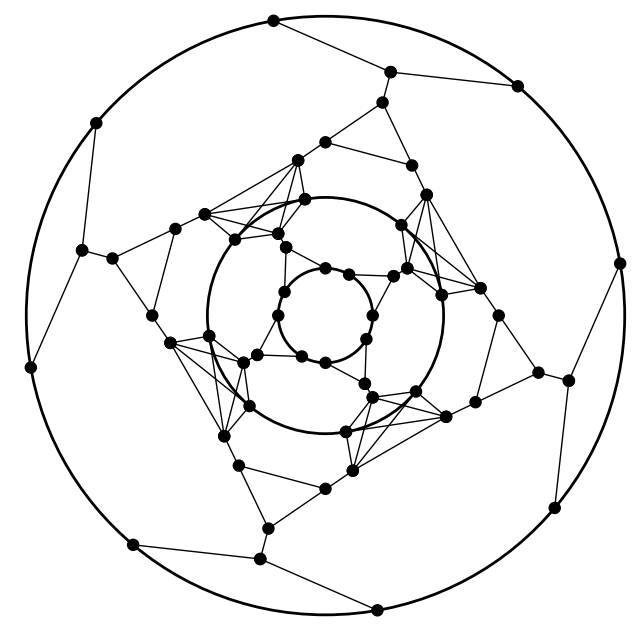

Figure 6. $L\left(S\left(Q_{8}\right)\right)$

\subsection{Convex polytope $R_{n}$}

The graph of convex polytope $R_{n}$ is obtained as a combination of the graph of a prism and the graph of antiprism as defined in [3]. The convex polytope $R_{n}$ for $n=8$ is shown in Figure 7.

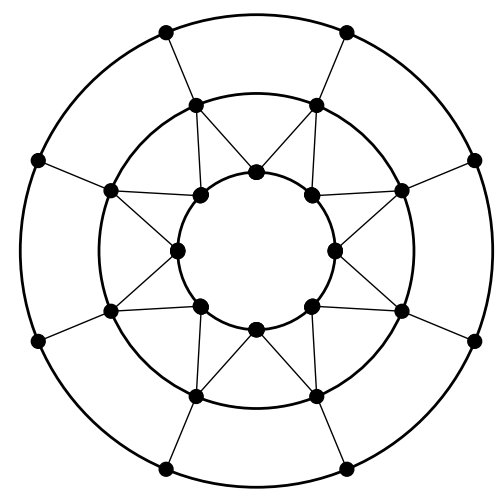


Figure 7. $R_{8}$

2.3.1. Subdivision of convex polytope $R_{n}$. We obtain the subdivision $S\left(R_{n}\right)$ by inserting additional vertex between each pair of adjacent vertices of $R_{n}$. The subdivision of $R_{n}$ for $n=8$ is shown in Figure 8. The $S\left(R_{n}\right)$ consists of $8 n$ vertices out which $6 n$ vertices are of degree $2, n$ vertices are of degree $3, n$ vertices are of degree 4 and $n$ vertices are of degree 5. Using Lemma 1.2 , we have $\left|E\left(S\left(R_{n}\right)\right)\right|=12 n$.

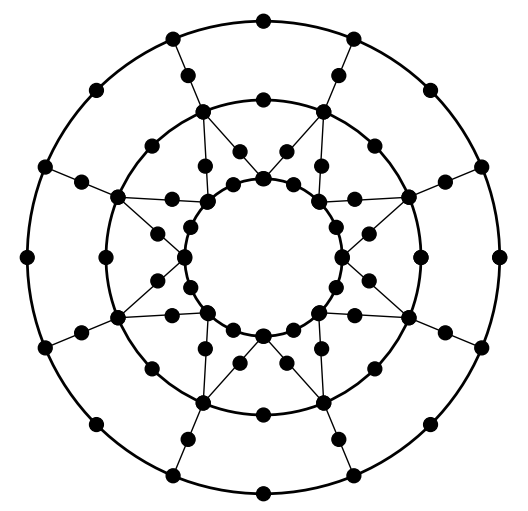

Figure 8. $S\left(R_{8}\right)$

2.3.2. Line graph of subdivision of convex polytope $R_{n}$. The line graph of subdivision of $R_{n}$ consists of $12 n$ vertices out which $3 n$ vertices are of degree $3,4 n$ vertices are of degree 4 and $5 n$ vertices are of degree 5. Using Lemma 1.2, we have $\left|E\left(L\left(S\left(R_{n}\right)\right)\right)\right|=25 n$. The $L\left(S\left(R_{n}\right)\right)$ is shown in Figure 9 for $n=8$.

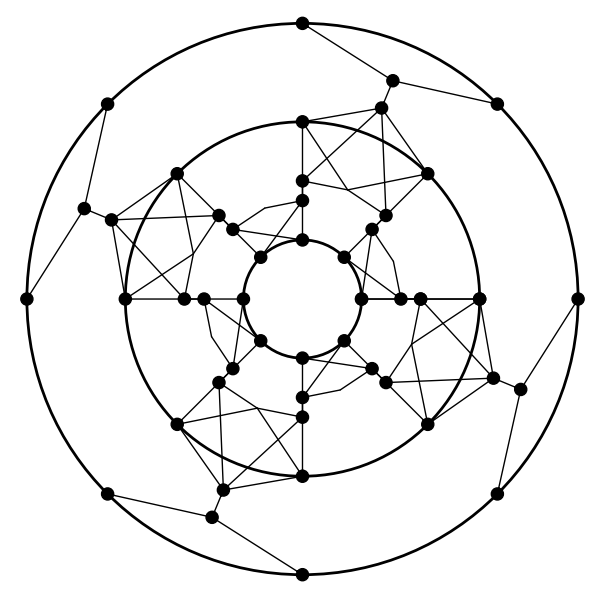

Figure 9. $L\left(S\left(R_{8}\right)\right)$

\subsection{The edge partition of line graphs of subdivision of convex polytopes}

For $u v \in E(G)$, let $d_{u}$ and $d_{v}$ are degrees of vertices $u$ and $v$ respectively. We partition $E(G)$ into subsets based on the degrees of the end vertices of edges in $G$. The edge partition of $L\left(S\left(D_{n}\right)\right), L\left(S\left(Q_{n}\right)\right)$ and $L\left(S\left(R_{n}\right)\right)$ with respect to degrees are shown in Tables 1,2 and 3 respectively.

\begin{tabular}{|c|c|}
\hline$\left(d_{u}, d_{v}\right)$ & Number of edges \\
\hline$(3,3)$ & $18 n$ \\
\hline
\end{tabular}

Table 1. The edge partition of $L\left(S\left(D_{n}\right)\right)$ 


\begin{tabular}{|c|c|}
\hline$\left(d_{u}, d_{v}\right)$ & Number of edges \\
\hline$(3,3)$ & $12 n$ \\
$(5,5)$ & $11 n$ \\
$(3,5)$ & $3 n$ \\
\hline
\end{tabular}

Table 2. The edge partition of $L\left(S\left(Q_{n}\right)\right)$

\begin{tabular}{|c|c|}
\hline$\left(d_{u}, d_{v}\right)$ & Number of edges \\
\hline$(3,3)$ & $4 n$ \\
$(3,5)$ & $n$ \\
$(5,5)$ & $11 n$ \\
$(4,5)$ & $2 n$ \\
$(4,4)$ & $7 n$ \\
\hline
\end{tabular}

Table 3. The edge partition of $L\left(S\left(R_{n}\right)\right)$

\section{Topological indices of line graph of subdivision of some convex poly- topes}

In this section we will discuss different types of topological indices and as an application, we compute the topological indices of the line graph of subdivision of some convex polytopes discussed in Section 2.

\subsection{First, second and third Zagreb indices}

Analyzing the structure-dependency of total $\pi$-electron energy, a pair of topological indices $M_{1}(G)$ and $M_{2}(G)$ were introduced known as Zagreb group indices (see [15]). Soon abbriviated to "Zagreb Index" and now a days "first Zagreb index" and "second Zagreb index" respectively. They provide quantative measures of molecular branching. They are defined as follows:

$$
\begin{aligned}
M_{1}(G) & =\sum_{u \in V(G)} d_{u}^{2} \\
M_{2}(G) & =\sum_{u v \in E(G)} d_{u} d_{v} .
\end{aligned}
$$

We can rewrite the first Zagreb index as

$$
M_{1}(G)=\sum_{u v \in E(G)}\left[d_{u}+d_{v}\right] .
$$

Some properties of first and second Zagreb indices can be viewed in $([6,12,16,19,30])$. In 1977, Alberton introduced the irregularity of graphs (see [1]). To confirm with the terminology of chemical graph theory Fath-Tabar (see [8]) called Alberton's irregularity the third Zagreb index and is defined as:

$$
M_{3}(G)=\sum_{u v \in E(G)}\left|d_{u}-d_{v}\right| .
$$

\subsection{Zagreb co-indices}

The first Zagreb coindex is defined as:

$$
\overline{M_{1}}(G)=\sum_{u v \notin E(G)}\left[d_{u}+d_{v}\right] .
$$


The second Zagreb coindex is defined as:

$$
\overline{M_{2}}(G)=\sum_{u v \notin E(G)} d_{u} d_{v} .
$$

Theorem 3.1. [10] Let $G$ be a graph of order $p$ and size $q$. Then

$$
\begin{aligned}
& M_{1}(\bar{G})=M_{1}(G)+p(p-1)^{2}-4 q(p-1) ; \\
& \overline{M_{1}}(G)=2 q(p-1)-M_{1}(G) ; \\
& \overline{M_{1}}(\bar{G})=2 q(p-1)-M_{1}(G) .
\end{aligned}
$$

Theorem 3.2. [17] Let $G$ be a graph of order $p$ and size $q$. Then

$$
\begin{aligned}
& M_{2}(\bar{G})=\frac{1}{2} p(p-1)^{3}-3 q(p-1)^{2}+2 q^{2}+\frac{2 p-3}{2} M_{1}(G)-M_{2}(G) ; \\
& \overline{M_{2}}(G)=2 q^{2}-\frac{1}{2} M_{1}(G)-M_{2}(G) ; \\
& \overline{M_{2}}(\bar{G})=q(p-1)^{2}-(p-1) M_{1}(G)+M_{2}(G) .
\end{aligned}
$$

\subsection{Hyper-Zagreb index}

The hyper-Zagreb index was first introduced in [27]. This index is defined as follows:

$$
H M(G)=\sum_{u v \in E(G)}\left(d_{u}+d_{v}\right)^{2} .
$$

\subsection{Randic index}

Randic index is the most studied and most popular among the all topological indices. It was introduced in 1975 (see [23]) by Milan Randic. He named it branching index, but nowadays it is known as Randic index. It is defined as:

$$
R(G)=\sum_{u v \in E(G)} \frac{1}{\sqrt{d_{u} d_{v}}}
$$

Later, this index was generalized by Bollobas and Erdös (see [5]) to the following form for any real number $\alpha$, and named the general Randic index:

$$
R(G)=\sum_{u v \in E(G)}\left[d_{u} d_{v}\right]^{\alpha} .
$$

\subsection{General sum-connectivity index}

The general sum-connectivity index $\chi(G)$ is a recent invention by B. Zhou and N. Trianjstic (see [31]). They replaced product of vertex degrees $d_{u} d_{v}$ by the sum $d_{u}+d_{v}$ in the Randic index. This index is defined as follows:

$$
\chi(G)=\sum_{u v \in E(G)} \frac{1}{\sqrt{d_{u}+d_{v}}} .
$$

\subsection{Geometric-arithmetic index}

D. Vukicevic and B. Furtula defined the first geometric arithmetic $G A$ index in [29]. This GA index for $G$ is defined as

$$
G A(G)=\sum_{u v \in E(G)} \frac{2 \sqrt{d_{u} d_{v}}}{d_{u}+d_{v}} .
$$




\subsection{Atom-bond connectivity index}

Ernesto Estrda et al. in [7] introduced a new topological index that is an amended version of Randic index. He named it "atom-bond connectivity index" and defined as

$$
A B C(G)=\sum_{u v \in E(G)} \sqrt{\frac{d_{u}+d_{v}-2}{d_{u} d_{v}}} .
$$

Theorem 3.3. Let $L\left(S\left(D_{n}\right)\right), L\left(S\left(Q_{n}\right)\right)$ and $L\left(S\left(R_{n}\right)\right)$ are the line graphs of subdivision of convex polytopes $D_{n}, Q_{n}$ and $R_{n}$ respectively then:

$M_{1}\left(L\left(S\left(D_{n}\right)\right)=108 n ; M_{2}\left(L\left(S\left(D_{n}\right)\right)=162 n ; M_{3}\left(L\left(S\left(D_{n}\right)\right)=0\right.\right.\right.$.

$M_{1}\left(L\left(S\left(Q_{n}\right)\right)=206 n ; M_{2}\left(L\left(S\left(Q_{n}\right)\right)=428 n ; M_{3}\left(L\left(S\left(Q_{n}\right)\right)=6 n\right.\right.\right.$.

$M_{1}\left(L\left(S\left(R_{n}\right)\right)=216 n ; M_{2}\left(L\left(S\left(R_{n}\right)\right)=478 n ; M_{3}\left(L\left(S\left(R_{n}\right)\right)=4 n\right.\right.\right.$.

Proof. The first, second and third Zagreb indices can be obtained by Formulas (3.1) (3.3) and using edge partitions as shown in Tables 1,2 and 3 respectively.

Theorem 3.4. Let $L\left(S\left(D_{n}\right)\right), L\left(S\left(Q_{n}\right)\right)$ and $L\left(S\left(R_{n}\right)\right)$ are the line graphs of subdivision of convex polytopes $D_{n}, Q_{n}$ and $R_{n}$ respectively then:

$M_{1}\left(\overline{\left(L\left(S\left(D_{n}\right)\right)\right.}\right)=1728 n^{3}-1152 n^{2}+192 n$.

$\overline{M_{1}}\left(\left(L\left(S\left(D_{n}\right)\right)\right)=432 n^{2}-144 n\right.$.

$M_{2}\left(\overline{\left(L\left(S\left(D_{n}\right)\right)\right.}\right)=10368 n^{4}-10368 n^{3}+3456 n^{2}-222 n-162$.

$\overline{M_{2}}\left(\left(L\left(S\left(D_{n}\right)\right)\right)=648 n^{2}-54 n-162\right.$.

$\overline{M_{2}}\left(\overline{L\left(S\left(D_{n}\right)\right)}\right)=2592 n^{3}-1728 n^{2}+126 n+162$.

$M_{1}\left(\overline{\left(L\left(S\left(Q_{n}\right)\right)\right.}\right)=2744 n^{3}-1848 n^{2}+324 n$.

$\overline{M_{1}}\left(\left(L\left(S\left(Q_{n}\right)\right)\right)=728 n^{2}-258 n\right.$.

$M_{2}\left(\overline{\left(L\left(S\left(Q_{n}\right)\right)\right.}\right)=19208 n^{4}-19404 n^{3}+6714 n^{2}-822 n$.

$\overline{M_{2}}\left(\left(L\left(S\left(Q_{n}\right)\right)\right)=1352 n^{2}-531 n\right.$.

$\overline{M_{2}}\left(\overline{L\left(S\left(Q_{n}\right)\right)}\right)=5096 n^{3}-3612 n^{2}+660 n$.

$M_{1}\left(\overline{\left(L\left(S\left(R_{n}\right)\right)\right.}\right)=1728 n^{3}-1488 n^{2}+328 n$.

$\overline{M_{1}}\left(\left(L\left(S\left(R_{n}\right)\right)\right)=600 n^{2}-266 n\right.$.

$M_{2}\left(\overline{\left(L\left(S\left(R_{n}\right)\right)\right.}\right)=10368 n^{4}-13392 n^{3}+5858 n^{2}-833 n$.

$\overline{M_{2}}\left(\left(L\left(S\left(R_{n}\right)\right)\right)=1250 n^{2}-536 n\right.$.

$\overline{M_{2}}\left(\overline{L\left(S\left(R_{n}\right)\right)}\right)=3600 n^{3}-3192 n^{2}+669 n$.

Proof. The Zagreb co-indices can be obtained by Theorems 3.1, 3.2 and using edge partitions as shown in Tables 1, 2 and 3 respectively.

Theorem 3.5. Let $L\left(S\left(D_{n}\right)\right), L\left(S\left(Q_{n}\right)\right)$ and $L\left(S\left(R_{n}\right)\right)$ are the line graphs of subdivision of convex polytopes $D_{n}, Q_{n}$ and $R_{n}$ respectively then:

$H M\left(L\left(S\left(D_{n}\right)\right)=648 n\right.$.

$H M\left(L\left(S\left(Q_{n}\right)\right)=1724 n\right.$.

$H M\left(L\left(S\left(R_{n}\right)\right)=1918 n\right.$.

Proof. The Hyper Zagreb index can be obtained by using Formula (3.10) and using edge partitions as shown in Tables 1, 2 and 3 respectively.

Theorem 3.6. Let $L\left(S\left(D_{n}\right)\right), L\left(S\left(Q_{n}\right)\right)$ and $L\left(S\left(R_{n}\right)\right)$ are the line graphs of subdivision of convex polytopes $D_{n}, Q_{n}$ and $R_{n}$ respectively then: 
$R\left(L\left(S\left(D_{n}\right)\right)=6 n\right.$.

$R\left(L\left(S\left(Q_{n}\right)\right)=\frac{31}{5} n+\frac{1}{5} n \sqrt{15}\right.$.

$R\left(L\left(S\left(R_{n}\right)\right)=\frac{317}{60} n+\frac{1}{15} n \sqrt{15}+\frac{1}{5} n \sqrt{5}\right.$.

Proof. The Randic index can be obtained by using Formula (3.11) and using edge partitions as shown in Tables 1, 2 and 3 respectively.

Theorem 3.7. Let $L\left(S\left(D_{n}\right)\right), L\left(S\left(Q_{n}\right)\right)$ and $L\left(S\left(R_{n}\right)\right)$ are the line graphs of subdivision of convex polytopes $D_{n}, Q_{n}$ and $R_{n}$ respectively then:

$\chi\left(\left(L\left(S\left(D_{n}\right)\right)\right)=3 n \sqrt{6}\right.$.

$\chi\left(\left(L\left(S\left(Q_{n}\right)\right)\right)=2 n \sqrt{6}+\frac{3}{4} n \sqrt{2}+\frac{11}{10} n \sqrt{10}\right.$.

$\chi\left(\left(L\left(S\left(R_{n}\right)\right)\right)=23 n \sqrt{6}+2 n \sqrt{2}+\frac{2}{3} n+\frac{11}{10} n \sqrt{10}\right.$.

Proof. The general sum-connectivity index $\chi(G)$ can be obtained by using Formula (3.13) and using edge partitions as shown in Tables 1, 2 and 3 respectively.

Theorem 3.8. Let $L\left(S\left(D_{n}\right)\right), L\left(S\left(Q_{n}\right)\right)$ and $L\left(S\left(R_{n}\right)\right)$ are the line graphs of subdivision of convex polytopes $D_{n}, Q_{n}$ and $R_{n}$ respectively then:

$G A\left(L\left(S\left(D_{n}\right)\right)=18 n\right.$

$G A\left(L\left(S\left(Q_{n}\right)\right)=23 n+\frac{3}{4} n \sqrt{15}\right.$.

$G A\left(L\left(S\left(R_{n}\right)\right)=22 n+\frac{1}{4} n \sqrt{1}+\frac{8}{9} n \sqrt{5}\right.$.

Proof. The geometric-arithmetic index can be obtained by using Formula (3.14) and using edge partitions as shown in Tables 1, 2 and 3 respectively.

Theorem 3.9. Let $L\left(S\left(D_{n}\right)\right), L\left(S\left(Q_{n}\right)\right)$ and $L\left(S\left(R_{n}\right)\right)$ are the line graphs of subdivision of convex polytopes $D_{n}, Q_{n}$ and $R_{n}$ respectively then:

$A B C\left(L\left(S\left(D_{n}\right)\right)=12 n\right.$.

$A B C\left(L\left(S\left(Q_{n}\right)\right)=8 n+\frac{3}{5} n \sqrt{10}+\frac{22}{5} n \sqrt{2}\right.$.

$A B C\left(L\left(S\left(R_{n}\right)\right)=\frac{8}{3} n+\frac{1}{5} n \sqrt{10}+\frac{7}{4} n \sqrt{6}+\frac{1}{5} n \sqrt{35}+\frac{22}{5} n \sqrt{2}\right.$.

Proof. The atom-bond Connectivity Index can be obtained by using Formula (3.15) and using edge partitions as shown in Tables 1,2 and 3 respectively.

\section{References}

[1] M.O. Alberton, The irregularity of a graph, Ars Combin. 46, 219-225, 1997.

[2] M. Baca, Labellings of two classes of convex polytopes, Util. Math. 34, 24-31, 1988.

[3] M. Baca, On magic labellings of convex polytopes, Ann. Discrete Math. 51, 13-16, 1992.

[4] S.H. Bertz, The bond graph, J. C. S. Chem. Commun. 818-820, 1981.

[5] B. Bollobas and P. Erdös, Graphs of extremal weights, Ars Combin. 50, 225-233, 1998. 
[6] K.C. Das and I. Gutman, Some properties of the second Zagreb index, MATCH Commun. Math. Comput. Chem. 52, 103-112, 2004.

[7] E. Estrada, L. Torres, L. Rodriguez and I. Gutman, An atom-bond connectivity index, Modelling the enthalpy of formation of alkanes, Indian J. Chem. 37, 849-855, 1998.

[8] G.H. Fath-Tabar, Old and new Zagreb indices of graphs, MATCH Commun. Math. Comput. Chem. 65, 79-84, 2011.

[9] B. Grünbaum, Graduate text in mathematics convex polytopes, Springer-Verlag, New York, 2003.

[10] I. Gutman, Selected properties of the schultz molecular topological index, J. Chem. Inf. Comput. Sci. 34, 1087-1089, 1994.

[11] I. Gutman, Edge versions of topological indices, in: Novel Molecular Structure Descriptors - Theory and Applications II , Univ. Kragujevac, Kragujevac, 2010.

[12] I. Gutman and K.C. Das, The first Zagreb index 30 years after, MATCH Commun. Math. Comput. Chem. 50, 83-92, 2004.

[13] I. Gutman and E. Estrada, Topological indices based on the line graph of the molecular graph, J. Chem. Inf. Comput. Sci. 36, 541-543, 1996.

[14] I. Gutman and Z. Tomovic, On the application of line graphs in quantitative structure-property studies, J. Serb. Chem. Soc. 65 (8), 577-580, 2000.

[15] I. Gutman and N. Trinajstic, Graph theory and molecular orbitals. Total $\pi$-electron energy of alternant hydrocarbons, Chem. Phys. Lett. 17, 535-538, 1972.

[16] I. Gutman, B. Furtula, A.A. Toropov and A.P. Toropova, The graph of atomic orbitals and its basic properties. 2. Zagreb indices, MATCH Commun. Math. Comput. Chem. 53, 225-230, 2005.

[17] I. Gutman, B. Furtula, Z.K. Vukićević and G. Popivoda, On Zagreb Indices and Coindices, MATCH Commun. Math. Comput. Chem. 74, 5-16, 2015.

[18] I. Gutman, L. Popovic, B.K. Mishra, M. Kaunar, E. Estrada and N. Guevara, Application of line graphs in physical chemistry. Predicting surface tension of alkanes, J. Serb. Chem. Soc. 62, 1025-1029, 1997.

[19] P. Hansen, H. Melot and I. Gutman, Variable neighborhood search for extremal graphs 12. A note on the variance of bounded degrees in graphs, MATCH Commun. Math. Comput. Chem. 54, 221-232, 2005.

[20] M. Imran, A.Q. Baig and A. Ahmed, Families of plane graphs with constant metric dimension, Util. Math. 88, 43-57, 2012.

[21] M. Imran, A.Q. Baig and M.K. Shafiq, Classes of convex polytopes with constant metric dimension, Util. Math. 90, 85-99, 2013.

[22] A. Iranmanesh, I. Gutman, O. Khormali and A. Mahmiani, The edge versions of the Wiener index, MATCH Comm. Math. Comput. Chem. 61, 663-672, 2009.

[23] M. Randic, On Characterization of Molecular Branching, J. Amer. Chem. Soc. 97, 6609-6615, 1975.

[24] M.F. Nadeem, S. Zafar and Z. Zahid, On certain Topological indices of the line graph of subdivision graphs, Appl. Math. Comput. 271, 790-794, 2015.

[25] M.F. Nadeem, S. Zafar and Z. Zahid, On Topological properties of the line graphs of subdivision graphs of certain nanostructures, Appl. Math. Comput. 273, 125-130, 2016.

[26] M.F. Nadeem, S. Zafar and Z. Zahid, Some Topological Indices of $L\left(S\left(C N C_{k}[n]\right)\right.$, Punjab Univ. J. Math. (Lahore), 49 (1), 13-17, 2017.

[27] G.H. Shirdel, H. Rezapour and A.M. Sayadi, The hyper-Zagreb index of graph operations, Iran. J. Math. Chem. 4 (2), 213-220, 2013.

[28] H. Van de Waterbeemd, R.E. Carter, G. Grassy, H. Kubiny, Y.C. Martin, M.S. Tutte, and P. Willet, Glossary of terms used in computational drug design, Pure Appl. Chem. 69, 1137-1152, 1997. 
[29] D. Vukicevic and B. Furtula, Topological index based on the ratios of geometrical and arithmetical means of end-vertex degrees of edges, J. Math. Chem. 46, 1369-1376, 2009.

[30] B. Zhou, Zagreb indices, MATCH Commun. Math. Comput. Chem. 52, 113-118, 2004.

[31] B. Zhou and N. Trinajstic, On general sum-connectivity index, J. Math. Chem. 47, 210-218, 2010. 